\title{
Taxocenose de bagres marinhos (Siluriformes, Ariidae) da região estuarina de São Vicente, $S P$, Brasil
}

\author{
Thassya Christina dos Santos Schmidt ${ }^{1,3,4}$, Itamar Alves Martins ${ }^{1}$, \\ Alvaro Luis Diogo Reigada ${ }^{2} \&$ June Ferraz Dias $^{3}$ \\ ${ }^{1}$ Laboratório de Zoologia, Universidade de Taubaté - UNITAU, \\ Av. Tiradentes, 500, CEP 12030-180, Taubaté, SP, Brasil \\ ${ }^{2}$ Universidade Metropolitana de Santos - UNIMES, \\ Rua da Constituição, 374, CEP 11015-140, Santos, SP, Brasil \\ ${ }^{3}$ Instituto Oceanográfico, Universidade de São Paulo - USP, \\ Praça do Oceanográfico, 191, CEP 05508-120, São Paulo, SP, Brasil \\ ${ }^{4}$ Autor para correspondência: Thassya Christina dos Santos Schmidt, e-mail: tcsschmidt@yahoo.com.br
}

SCHMIDT, T.C.S., MARTINS, I.A., REIGADA, A.L.D. \& DIAS, J.F. 2008. Taxocenose of marine catfish (Siluriformes, Ariidae) in the complex bay-estuary of São Vicente, SP, Brazil. Biota Neotrop. 8(4): http:// www.biotaneotropica.org.br/v8n4/en/abstract?article+bn01108042008.

\begin{abstract}
The purpose of this study was to analyze the taxocenose of marine catfish in the complex bay-estuary of Sao Vicente, Brazil. The sampling was done monthly from September/2000 to August/2002, in four places of the estuary. Were caught 3.985 individuals belonging for 5 genus and 6 species, totalizing $47.807 \mathrm{~g}$. The specie Cathorops spixii showed the biggest plentiful with 1.569 individuals sampled. In the transect I (close to the coastal region), it was registered the biggest richness (6 species) and in the transect IV (upper estuary) the biggest number of individuals $(n=2.602)$ e weight of $26.236 \mathrm{~g}$. In March/2002 were registered the biggest occurrence with 687 individuals and biggest weight in February/2002 (8.816 g). The bottom water salinity, in the time of study, changed between 14 and 36, and the water temperature varied between 19 and $30{ }^{\circ} \mathrm{C}$. In the sample place juveniles and adults' individuals were caught, a mostly juvenile which demonstrates that of this place shows favorable conditions for growth.

Keywords: spatial and temporal distribution, family Ariidae, population structure, Complex bay-estuary of Sao Vicente.
\end{abstract}

SCHMIDT, T.C.S., MARTINS, I.A., REIGADA, A.L.D. \& DIAS, J.F. 2008. Taxocenose de bagres marinhos (Siluriformes, Ariidae) da região estuarina de São Vicente, SP, Brasil. Biota Neotrop. 8(4): http://www. biotaneotropica.org.br/v8n4/pt/abstract?article+bn01108042008.

Resumo: O objetivo do trabalho foi analisar a taxocenose de bagres marinhos do complexo baía-estuário de São Vicente, SP. As coletas foram mensais, no período de setembro de 2000 a agosto de 2002, em quatro regiões do estuário. Foram capturados 3.985 exemplares, pertencentes a cinco gêneros e seis espécies, totalizando $47.807 \mathrm{~g}$. A espécie Cathorops spixii apresentou a maior abundância numérica com 1.569 indivíduos amostrados. No transecto I (próximo da região costeira) foi registrada a maior riqueza e no transecto IV (estuário acima) a maior abundância com 2.602 exemplares e peso de 26.236 g. Em março de 2002 registrou-se a maior abundância com 687 indivíduos e o maior peso ocorreu em fevereiro de 2002 (8.816 g). A salinidade da água de fundo, ao longo do período de estudo, variou entre 14 e 36 e a temperatura da água variou entre 19 e $30^{\circ} \mathrm{C}$. Na região amostrada foram capturados exemplares jovens e adultos, ocorrendo um predomínio de jovens, o que demonstra que esta área apresenta condições favoráveis para crescimento.

Palavras-chave: distribuição espaço-temporal, família Ariidae, estrutura populacional, Complexo baía-estuário de São Vicente. 


\section{Introdução}

Os bagres ocorrem nas zonas litorâneas tropicais e subtropicais, em ambientes marinhos, estuarinos e em águas interiores, sendo geralmente mais abundantes em águas costeiras pouco profundas, em fundo lodoso ou arenoso (Araújo 1988, Andreata et al. 1989). Espécies exclusivamente marinhas podem ser encontradas em profundidades maiores que $100 \mathrm{~m}$ (Marceniuk 2005).

As regiões estuarinas e costeiras são consideradas locais de alimentação, reprodução e abrigo para estas espécies (Gurgel et al. 2000) e durante o período de desova, muitas espécies de bagres marinhos normalmente deslocam-se para a desembocadura dos rios e regiões lagunares (Figueiredo \& Menezes 1978).

Para o litoral brasileiro é relatada a ocorrência de oito gêneros e 21 espécies, e para a região sudeste, sete gêneros e 10 espécies (Aspistor luniscutis, Bagre bagre, B. marinus, Cathorops spixii, Genidens barbus, G. genidens, G. machadoi, Hexanematichthys parkeli, Notarius grandicassis e Potamarius grandoculis) (Figueiredo \& Menezes 1978, Menezes et al. 2003).

Trabalhos referentes aos aspectos biológicos e ecológicos dos bagres marinhos no litoral brasileiro são comuns. Com relação à ocorrência e distribuição, Arius spixii (=Cathorops spixii), Sciadeichthys luniscutis (=Aspistor luniscutis) e Netuma barba (=Genidens barbus) ocorrem em maiores profundidades, enquanto Genidens genidens em áreas mais rasas (Mishima \& Tanji 1981), sendo que a salinidade parece condicionar a ocorrência das espécies (Mishima \& Tanji 1983a). Em termos de abundância, Netuma barba (=G. barbus) é a espécie mais abundante no estuário da Lagoa dos Patos (Araújo 1988), enquanto Cathorops spixii foi a mais abundante em Peruíbe (Craig 1980), em Cananéia (Mishima \& Tanji 1983a) e no complexo Mundaú/Manguaba (Melo \& Teixeira 1992), e Genidens genidens na baía de Sepetiba (Azevedo et al. 1998). As diferentes espécies de bagre apresentam amplo espectro alimentar, sendo constatado que adultos de espécies que atingem tamanhos menores e juvenis de espécies de grande porte mostram variação qualitativa na dieta, embora vivam em mesmo ambiente (Mishima \& Tanji 1982). A dieta é constituída principalmente por detrito, decápodes, peixes, poliquetos e bivalves (Mishima \& Tanji 1982, Araújo 1984, Espírito Santo \& Isaac 1999). Machos apresentam menor peso em mesmo comprimento que as fêmeas como possível resposta à incubação oral dos machos e a parada na alimentação durante este período (Araújo et al. 1998b).

Os bagres apresentam deslocamentos sazonais e relacionados às fases do ciclo de vida (Craig 1980, Mishima \& Tanji 1981, 1983b, Reis 1986, Araújo 1988, Araújo et al. 1998b, Azevedo et al. 1998, 1999). A reprodução ocorre em diferentes épocas, mas principalmente relacionada aos meses mais quentes ou com maior aporte de águas interiores: do final da primavera ao início do verão para Genidens genidens em Jacarepaguá, Sepetiba e Maricá (Rio de Janeiro), Cananéia (São Paulo) e Guaratuba (Paraná) (Mishima \& Tanji 1983b, Barbieri et al. 1992, Chaves 1994, Araújo et al. 1998a, Silva et al. 1998, Gomes et al. 1999, Mazzoni et al. 2000, Gomes \& Araújo 2004); na primavera e início do verão para Cathorops spixii em Sepetiba, Cananéia e baía dos Pinheiros (Paraná) (Mishima \& Tanji 1983b, Gomes et al. 1999, Fávaro et al. 2005), e no verão e início do outono em Natal (Rio Grande do Norte) (Gurgel et al. 2000); na primavera para Sciadeichthys luniscutis (=A. luniscutis) em Sepetiba (Gomes \& Araújo 2004) e na primavera e verão em Cananéia (Mishima \& Tanji 1983a); no outono para Bagre marinus na costa de Pernambuco (Pinheiro et al. 2006); e no final da primavera e início do verão para Netuma barba (=G. barbus) no estuário da Lagoa dos Patos (Rio Grande do Sul) (Reis 1986).

O desenvolvimento ovocitário é sincrônico, sugerindo desova total (Araújo et al. 1998a, Silva et al. 1998), embora Gurgel et al. (2000) afirmem que a desova seja parcelada para C. spixii, baseados unicamente na extensão do período de desova, desconsiderando, porém, o aspecto populacional nessa extensão. A incubação ocorre no verão para Genidens genidens e há indicação de alimentação exógena das larvas durante a incubação (Chaves 1994), com duração de dois a três meses (Barbieri et al. 1992). De maneira geral, a fecundidade é baixa e varia com as espécies, entre um mínimo de 7 e máximo de 38 ovócitos para G. genidens (Barbieri et al. 1992, Araújo et al. 1998a, Gomes et al. 1999), de 11 a 22 para B. marinus (Pinheiro et al. 2006), entre 6 e 24 para Sciadeichthys luniscutis (=A. luniscutis) (Gomes \& Araújo 2004), entre 32 e 272 para Netuma barba (=G. barbus) (Reis 1986). O cuidado com a prole, aliado às baixas fecundidades, podem levar a uma reposição lenta dos estoques (Velasco et al. 2007).

Apesar do conhecimento acumulado, não há relato de estudos sobre as espécies de Ariidae na região estuarina de São Vicente. Assim, o objetivo deste trabalho é descrever a estrutura da comunidade de bagres marinhos em relação a sua distribuição espaço-temporal, além de alguns aspectos da estrutura populacional das espécies mais abundantes amostradas no estuário de São Vicente.

\section{Material e Métodos}

As coletas foram mensais, no período entre setembro de 2000 e agosto de 2002, realizadas na região estuarina do município de São Vicente $\left(23^{\circ} 55^{\prime}\right.$ a $24^{\circ} 00^{\prime} \mathrm{S}$ e $46^{\circ} 21^{\prime}$ a $\left.46^{\circ} 30^{\prime} \mathrm{W}\right)$ (Figura 1$)$.

Foram estabelecidas quatro áreas, em diferentes locais do estuário denominadas de transectos (TI, TII, TIII e TIV) (Figura 1), sendo que para cada local em cada mês foram tomados os dados de temperatura da água de fundo com um termômetro de mercúrio $\left({ }^{\circ} \mathrm{C}\right)$ e a salinidade da água por meio de um refratômetro óptico. O transecto I corresponde à região mais externa amostrada, com uma profundidade média de $5 \mathrm{~m}$, o transecto II está localizado em frente da praia Paranapuã, com uma profundidade média de $4 \mathrm{~m}$, o transecto III localiza-se no largo de São Vicente, com uma profundidade média de $4 \mathrm{~m}$ e o transecto IV corresponde a área mais interna do estuário, no largo de São Vicente, com uma profundidade média de $6 \mathrm{~m}$ (Figura 1).

Para a captura dos exemplares foi utilizada uma rede de arrasto de fundo do tipo "otter-trawl", de 7,5 m de comprimento com $15 \mathrm{~mm}$ entre nós na panagem e $10 \mathrm{~mm}$ no saco, com abertura de boca de 3,7 m de largura e $2 \mathrm{~m}$ de altura, operada de um barco de alumínio, cada arrasto teve duração média de 30 minutos. Os exemplares capturados foram mantidos congelados e, em laboratório, os bagres foram separa-

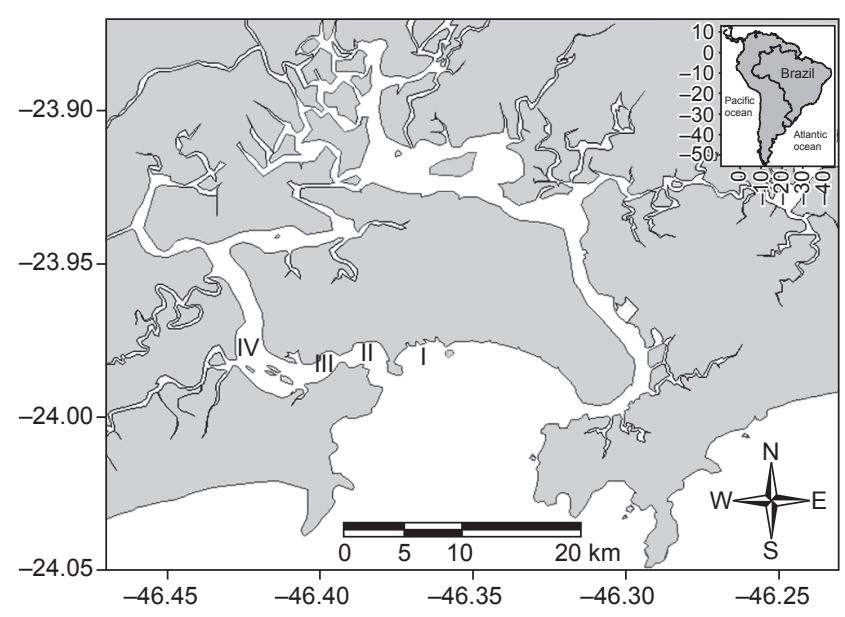

Figura 1. Localização dos locais de coleta.

Figure 1. Location of the sampled area. 
dos e identificados, utilizando-se o manual de Figueiredo \& Menezes (1978). A nomenclatura dos gêneros e espécies foi atualizada a partir de literatura pertinente (Menezes et al. 2003, Marceniuk 2005).

Foram calculados o número e o peso dos indivíduos por arrasto de pesca amostrados em cada transecto. Para a riqueza foi considerada o número de espécies e abundância em cada transecto $(\mathrm{R}=(\mathrm{S}-1) / \log \mathrm{N})$ e os valores de diversidade foram estimados pelo índice de diversidade de Shannon $\left(\mathrm{H}^{\prime}=-\sum(\mathrm{pi} * \log \mathrm{pi})\right)$.

A constância de ocorrência das espécies foi estimada por $\mathrm{C}=$ pi $* 100 * \mathrm{P}^{-1}$, sendo $\mathrm{C}=$ constância de ocorrência, pi = número de coletas em que a espécie i apareceu, $\mathrm{P}=$ número total de coletas. As espécies foram classificadas de acordo com os valores estimados: espécies constantes para $C \geq 50$, espécies acessórias para $25>C<50$, espécies acidentais para $C \leq 25$ (Dajoz 1973). A similaridade da abundância total entre os meses de coleta foi avaliada pela análise de agrupamento (índice de similaridade de Bray-Curtis) e a abundância das espécies por transecto foi testada pela análise de variância (ANOVA - não paramétrica - Kruskal-Wallis). Possíveis correlações entre os fatores abióticos (temperatura e salinidade da água de fundo) e a ocorrência (abundância numérica) dos exemplares, foram avaliadas pelo coeficiente de correlação de Spearman $\left(\mathrm{r}_{\mathrm{s}}\right)$, com nível de significância de 5\% (Ayres et al. 2003).

Para os espécimes capturados foram tomadas as medidas de comprimento padrão $(\mathrm{mm})$ e peso total $(\mathrm{g})$ (precisão de $0,1 \mathrm{~g}$ ). Os exemplares-testemunho foram depositados na Coleção Científica do Laboratório de Zoologia da Universidade de Taubaté, São Paulo.

\section{Resultados}

As variáveis ambientais medidas apresentaram variações sazonais, principalmente a salinidade. No transecto I a salinidade apresentou variação entre 28 e 36 . Nas áreas mais internas do estuário (transecto III e IV), bem como no transecto II, houve queda brusca na salinidade, principalmente em dezembro de 2000 , quando a menor salinidade foi registrada (14) no transecto III (Figura 2). O padrão de variação da temperatura da água de fundo para todas as áreas durante todo período de coletas, mostrou oscilações entre os 19 e $30^{\circ} \mathrm{C}$, com os valores mais baixos nos meses de outono e inverno, e aumento da temperatura nos meses da primavera e verão de ambos os anos. Para os quatro transectos amostrados, as maiores temperaturas foram registradas em fevereiro de 2001 e março de 2002 (Figura 2).

Foram amostrados 3.985 exemplares, totalizando $47.807 \mathrm{~g}$, representados por cinco gêneros e seis espécies: Cathorops spixii (Agassiz, 1829), Genidens genidens (Valenciennes, 1840), Aspistor luniscutis (Valenciennes, 1840), Genidens barbus (Lacepède, 1803), Notarius grandicassis (Valenciennes, 1840) e Bagre bagre (Linnaeus, 1758) (Tabela 1). C. spixii, G. genidens e A. luniscutis representaram mais de $87 \%$ da captura em número e em peso.

Em termos espaciais, no transecto I foi registrada a maior riqueza, com seis espécies, enquanto que nos transectos II, III e IV foram capturadas cinco espécies em cada. O transecto IV apresentou maior abundância $(65,3 \%)$ e peso total $(54,9 \%)$ e no transecto III ocorreu a menor abundância e o menor peso: 2,1 e $2,8 \%$, respectivamente (Tabela 2). A maior diversidade específica para a família Ariidae no estuário de São Vicente foi observada no transecto IV, seguida dos transectos III, I e II (Tabela 2).

O transecto IV, o mais interno no estuário, foi o local amostrado com os maiores valores de constância de ocorrência das espécies. Os resultados indicam $C$. spixii como constante nos transectos I e IV (58,3 e 50\%, respectivamente), acessória no transecto II e acidental no transecto III (Tabela 3). As espécies G. genidens e A. luniscutis foram constantes nas amostragens do transecto IV (75\% e 54,2\%, respectivamente) e G. barbus foi classificada como de ocorrência acessória nos transectos I e IV (33,3\% e 45,8\%, respectivamente). As espécies $N$. grandicassis e B. bagre foram acidentais em todos os transectos amostrados (Tabela 3).

Tabela 1. Espécies amostradas da família Ariidae no estuário de São Vicente, SP. ( $\mathrm{N}=$ número de exemplares, \% = freqüência relativa, peso (g) e $\mathrm{P}(\%)=$ porcentagem do peso de cada espécie amostrada.

Table 1. Species caught of the Ariidae family in complex bay-estuary of São Vicente, SP, Brazil ( $\mathrm{N}=$ number of species, \% = relative frequency, weight (g) and $\mathrm{W}(\%)=$ percentage of the weight of each specie caught.

\begin{tabular}{lrrrr}
\hline \multicolumn{1}{c}{ Espécies } & $\mathbf{N}$ & $\mathbf{( \% )}$ & Peso $(\mathbf{g})$ & P $(\boldsymbol{\%})$ \\
\hline Cathorops spixii (Agassiz, 1829) & 1569 & 39,4 & 18.924 & 39,6 \\
Genidens genidens (Valenciennes, 1840) & 1108 & 27,8 & 9.644 \\
Aspistor luniscutis (Valenciennes, 1840) & 808 & 20,3 & 12.734 \\
Genidens barbus (Lacepède, 1803) & 451 & 11,3 & 2.935 & 26,2 \\
Notarius grandicassis (Valenciennes, 1840) & 47 & 1,2 & 2.920 \\
Bagre bagre (Linnaeus, 1758) & 2 & 0,1 & 0.65 \\
\hline
\end{tabular}

Tabela 2. Número de exemplares, peso e suas respectivas freqüências relativas N (\%) e P (\%), diversidade de Shannon (H'), número de indivíduos por arrasto de pesca e peso dos indivíduos por arrasto de pesca para cada transecto amostrado.

Table 2. Number of individuals, weight and the relative frequency of the number N (\%) and W (\%), Shannon diversity (H'), number and weight of individuals for each fishery sample for each transect.

\begin{tabular}{|c|c|c|c|c|c|c|c|}
\hline & $\mathbf{N}$ & $\mathbf{N}(\%)$ & Peso (g) & $\mathbf{P}(\%)$ & Diversidade & $\begin{array}{c}\text { Número de indivíduos por } \\
\text { arrasto de pesca }\end{array}$ & $\begin{array}{l}\text { Peso dos indivíduos por } \\
\text { arrasto de pesca }\end{array}$ \\
\hline Transecto I & 473 & 11,9 & 4.234 & 8,9 & 1,28 & 15,77 & 0,141 \\
\hline Transecto II & 825 & 20,7 & 16.014 & 33,5 & 0,90 & 27,50 & 0,534 \\
\hline Transecto III & 85 & 2,1 & 1.323 & 2,8 & 1,34 & 2,83 & 0,044 \\
\hline Transecto IV & 2602 & 65,3 & 26.236 & 54,9 & 1,36 & 86,73 & 0,875 \\
\hline
\end{tabular}




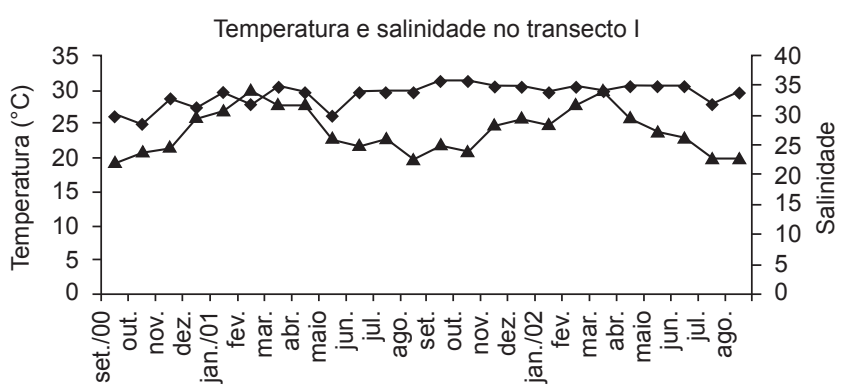

Meses amostrados

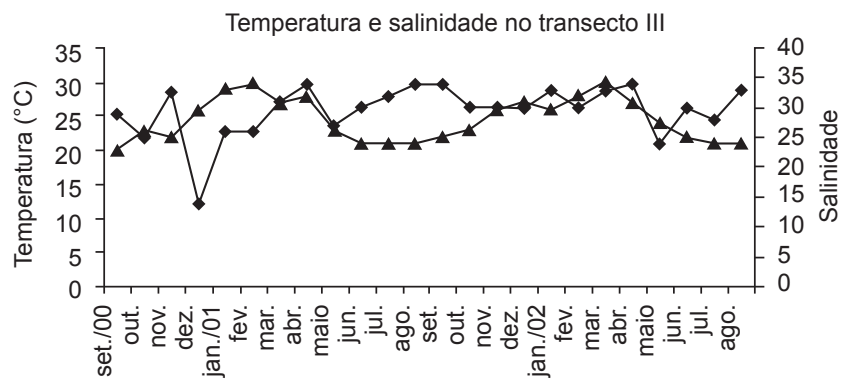

Meses amostrados

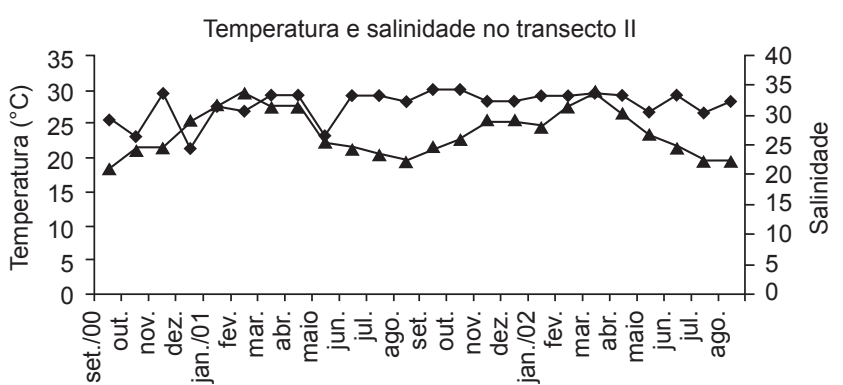

Meses amostrados

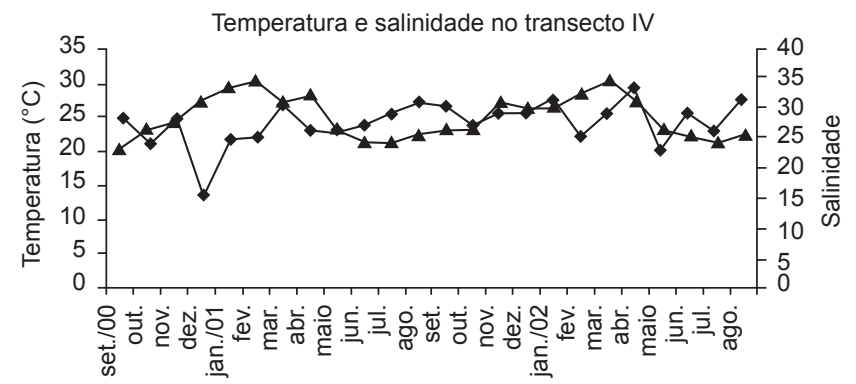

Meses amostrados

\section{$\multimap$ Temperatura $\prec$ Salinidade}

Figura 2. Temperatura e salinidade da água de cada transecto de amostragem no estuário de São Vicente.

Figure 2. Temperature and salinity of the water in each sample transect in the São Vicente estuary.

Tabela 3. Constância de ocorrência (C), freqüência relativa em termos de abundância (\%) e peso (P\%) por espécie em cada transecto amostrado na região estuarina de São Vicente, SP. (CASP = Cathorops spixii, GEGE = Genidens genidens, ASLU = Aspistor luniscutis, GEBA = Genidens barbus, NOGR = Notarius grandicassis, $\mathrm{BABA}=$ Bagre bagre, $\mathrm{T}=$ transecto de amostragem).

Table 3. Occurrence constancy (C), relative frequency in abundance (\%) and weight (W\%) for specie in each transect of the complex bay-estuary of São Vicente, SP. (CASP = Cathorops spixii, GEGE = Genidens genidens, ASLU = Aspistor luniscutis, GEBA= Genidens barbus, NOGR = Notarius grandicassis, $\mathrm{BABA}=$ Bagre bagre, $\mathrm{T}=$ transect $)$.

\begin{tabular}{|c|c|c|c|c|c|c|c|c|c|c|c|c|}
\hline \multicolumn{13}{|c|}{ Constância de ocorrência } \\
\hline \multirow[t]{2}{*}{ Espécie } & \multicolumn{3}{|c|}{$\mathbf{T} \mathbf{I}$} & \multicolumn{3}{|c|}{ T II } & \multicolumn{3}{|c|}{ T III } & \multicolumn{3}{|c|}{ T IV } \\
\hline & $\mathbf{C}$ & $(\%)$ & $\mathbf{P}(\%)$ & $\mathbf{C}$ & $(\%)$ & $\mathbf{P}(\%)$ & $\mathbf{C}$ & $(\%)$ & $\mathbf{P}(\%)$ & $\mathbf{C}$ & $(\%)$ & $\mathbf{P}(\%)$ \\
\hline CASP & 58,3 & 41,6 & 26,9 & 39,5 & 73,2 & 33,1 & 8,3 & 23,5 & 11,3 & 50,0 & 28,7 & 47,0 \\
\hline GEGE & 29,2 & 30,9 & 36,7 & 12,5 & 9,6 & 0,7 & 37,5 & 27,1 & 56,4 & 75,0 & 33,1 & 27,5 \\
\hline ASLU & 41,7 & 20,1 & 12,8 & 33,3 & 11,5 & 48,6 & 12,5 & 9,4 & 7,3 & 54,2 & 23,4 & 16,5 \\
\hline GEBA & 33,3 & 6,3 & 7,8 & 4,2 & 1,0 & 0,1 & 25,0 & 38,8 & 22,3 & 45,8 & 14,6 & 8,7 \\
\hline NOGR & 4,2 & 0,6 & 0,5 & 4,2 & 4,7 & 17,5 & 4,2 & 1,2 & 2,7 & 4,2 & 0,2 & 0,2 \\
\hline BABA & 4,2 & 0,4 & 15,4 & - & - & - & - & - & - & - & - & - \\
\hline
\end{tabular}

Do ponto de vista temporal, a maior riqueza numérica foi registrada em setembro de 2000 com seis espécies, e no mês de outubro de 2000 foi capturada apenas Genidens genidens. A maior abundância de exemplares foi registrada em março de 2002 com 687 indivíduos $(17,3 \%)$ e a maior massa em fevereiro de $2002 \mathrm{com} 8.816 \mathrm{~g}(18,6 \%)$. Os meses de setembro de 2001 e janeiro de 2002 foram os menos representativos com cinco exemplares cada, sendo que em janeiro de 2002, foi registrado o menor peso (4 g) (Figuras 3 e 4).

Somente no mês de setembro de 2000 foram capturadas todas as espécies, sendo que $A$. luniscutis apresentou maior frequiência do total de exemplares amostrados (50\%), seguida por $N$. grandicassis
(35,8\%), C. spixii (7,5\%), G. genidens (3,5\%), G. barbus e B. bagre ( $1,6 \%$ cada), sendo que esta última foi amostrada somente neste mês (Figura 3). A espécie G. genidens apresentou maior frequiência nos meses de verão e outono, em relação às outras espécies, enquanto para C. spixii, as maiores freqüências ocorreram na primavera, verão e inverno, apesar de sua ampla distribuição. A maior ocorrência de A. luniscutis, com 495 indivíduos capturados (72\%), foi registrada em março de 2002 (Figura 3).

Com relação ao peso, as maiores frequiências de $C$. spixii foram amostradas em dezembro de 2000 (49,8\%), fevereiro $(69,9 \%)$, abril (45\%), junho $(81,7 \%)$, agosto e setembro $(84 \%, 60,2 \%$, respectiva- 


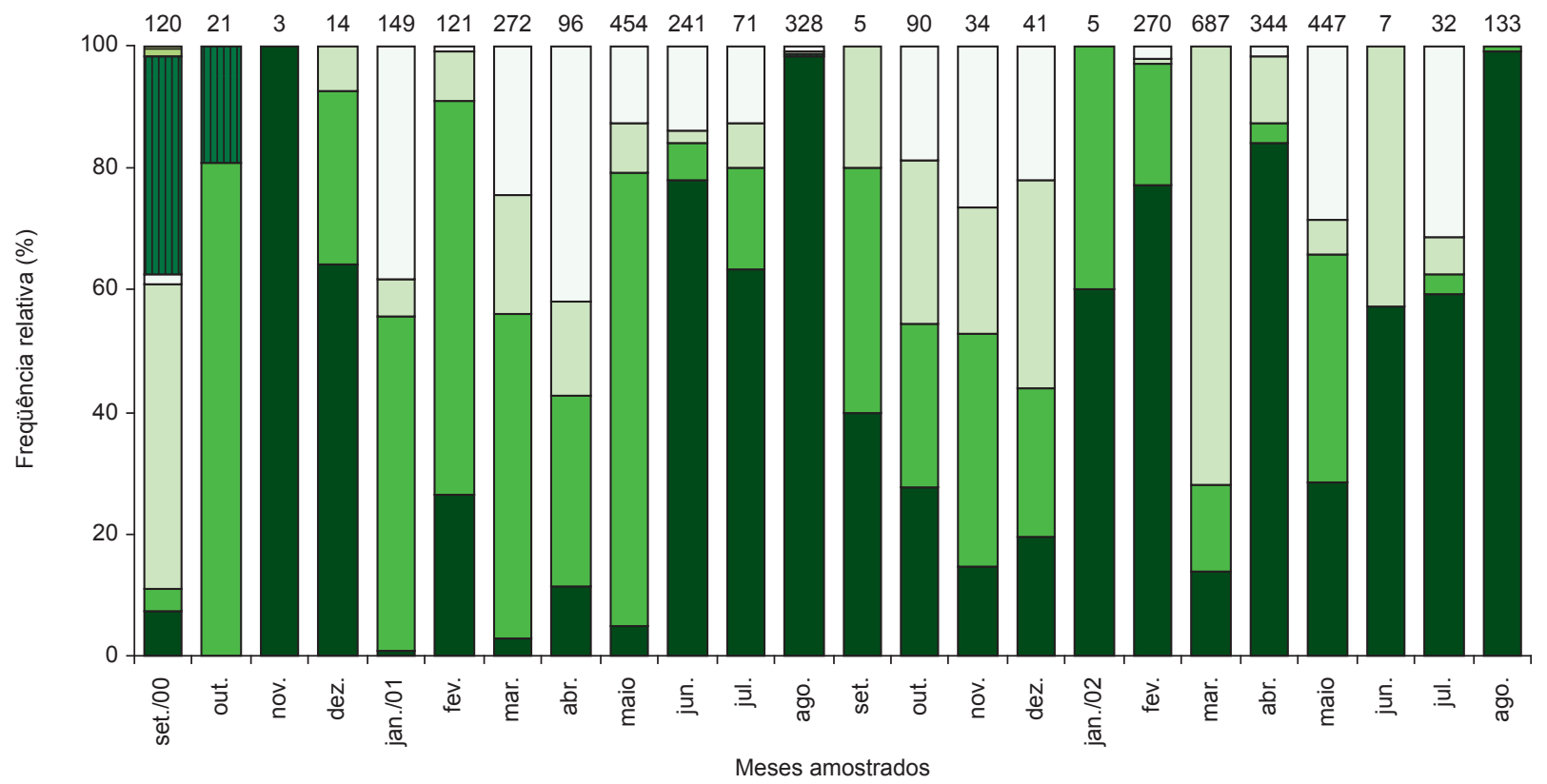

C.spixii $\square$ G.genidens $\square$ A. luniscutis $\square$ G. barbus $\square$ N.grandicassis $\quad$ 目 B. bagre

Figura 3. Frequiência relativa do número de exemplares de cada espécie de bagre marinho capturado ao longo de cada mês amostrado na região estuarina de São Vicente, SP. Os números posicionados acima das barras correspondem ao número total amostrado em cada mês

Figure 3. Relative frequency of individuals number for each species of the marine catfish sampled monthly in the São Vicente. The above number of each bar represents the total number sampled in each month.

mente) de 2001, fevereiro (87,8\%), abril $(81,1 \%)$, julho $(51,3 \%)$ e agosto $(98,9 \%)$ de 2002 , enquanto que para Genidens genidens as maiores freqüências ocorreram em janeiro $(59,8 \%)$, março $(64,5 \%)$, maio $(68,5 \%)$, julho $(59,8 \%)$, novembro $(45,2 \%)$ de 2001 e maio de 2002 ( 49,5\%) (Figura 4).

A análise de agrupamento revelou maior similaridade entre os meses de novembro e dezembro de 2001 e entre março e maio de 2001 (Figura 5), podendo-se observar que alguns meses do ano de 2001 reuniram-se num agrupamento maior, com nível de similaridade pouco menor.

Não houve correlação entre a salinidade da água de fundo e o número de exemplares amostrados em todos os transectos nem com a ocorrência das espécies, o mesmo ocorrendo em relação à temperatura e o número de exemplares amostrados ao longo dos meses nos transectos I, II e III. Entretanto, para o transecto IV houve correlação positiva e significativa $\left(r_{s}=0,41, t=2,15, p=0,04\right)$ entre a temperatura da água de fundo e o número de exemplares. No transecto IV A. luniscutis apresentou correlação com a temperatura ( $\mathrm{p}>0,05)$. Com relação às espécies, houve diferenças no transecto IV para G. genidens e C. spixii no transecto III $(\mathrm{p}=0,06)$.

Com relação à dinâmica populacional, o comprimento padrão das espécies capturadas variou entre 28 e $265 \mathrm{~mm}$, sendo que Cathorops spixii apresentou amplitude de comprimento padrão variando entre 30 e $195 \mathrm{~mm}(83,4 \pm 27,5 \mathrm{~mm})$, Genidens genidens entre 28 e $227 \mathrm{~mm}(88 \pm 34,8 \mathrm{~mm})$, A. luniscutis entre 33 e $265 \mathrm{~mm}$ (102 $\pm 51,4 \mathrm{~mm})$, Genidens barbus entre 35 e 196mm ( $80 \pm 18,2 \mathrm{~mm})$ e para Notarius grandicassis, o comprimento variou entre 84 e $234 \mathrm{~mm}$ (106 $\pm 33,3 \mathrm{~mm}$ ) (Figura 6). Foram capturados somente dois exemplares, com comprimento padrão de 137 e $150 \mathrm{~mm}$, da espécie Bagre bagre.

\section{Discussão}

As espécies A. luniscutis, B. bagre, C. spixii, G. barbus, G. genidens e N. grandicassis, capturadas na região estuarina de São Vicente, são consideradas comuns no litoral brasileiro, ocorrendo em grande quantidade em estuários e lagoas estuarinas (Figueiredo \& Menezes 1978). Segundo Ribeiro Neto (1993) quatro espécies figuram entre as mais importantes do complexo baía-estuário de Santos e São Vicente, sendo C. spixii a mais abundante nos fundos menos estruturados (areia e lama) da baía de Santos, Genidens barbus mostrando-se mais abundante nos ambientes de praia, e G. genidens e A. luniscutis mais comuns nos ambientes mais estruturados (fundo biogênico e parcéis) da baía.

As espécies capturadas no presente estudo foram registradas em outros trabalhos em diferentes regiões, tais como em Ubatuba (Rocha 1990, Cunningham 1983, Maciel 1995), em Santos e São Vicente (Paiva-Filho et al. 1987), e em Cananéia (Mishima \& Tanji 1981, ZaniTeixeira 1983). As capturas na região de Ubatuba reforçam a idéia de que estas espécies não podem ser consideradas estuarino-dependentes. Em outras áreas também foram amostrados exemplares de bagres marinhos, porém com somente algumas espécies capturadas, como na Baía de Sepetiba por Araújo et al. (1998a), Azevedo et al. (1999) e Pessanha et al. (2000), em Santos (Ribeiro Neto 1989), em Peruíbe (Craig 1980) e em Cananéia (Saul 1994).

As maiores freqüências numéricas ocorreram na região interna do estuário de São Vicente (transecto IV), podendo ser um indicativo da preferência destas espécies por locais estuarinos mais protegidos. Segundo Azevedo et al. (1999), esta preferência indica uma estratégia de coexistência desse grupo de peixes, que utilizam ambientes costeiros tropicais semi-abertos, com maiores abundâncias nas zonas com menores profundidades, transparência e salinidade. 


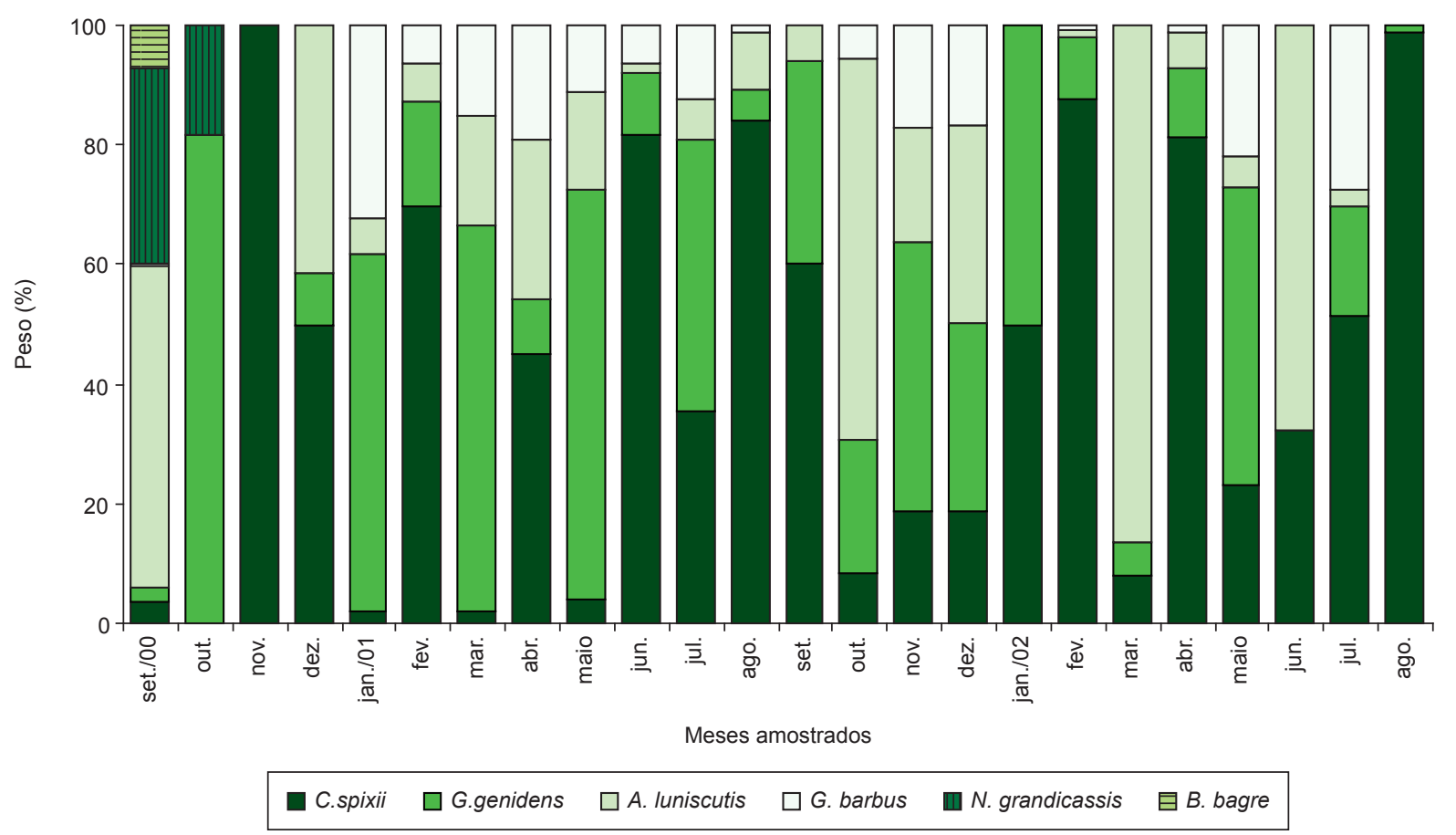

Figura 4. Frequiência relativa do peso de cada espécie de bagre marinho capturado, ao longo de cada mês amostrado na região estuarina de São Vicente, SP. Figure 4. Relative frequency of weight for each marine catfish species caught monthly in the complex bay-estuary of São Vicente, SP.

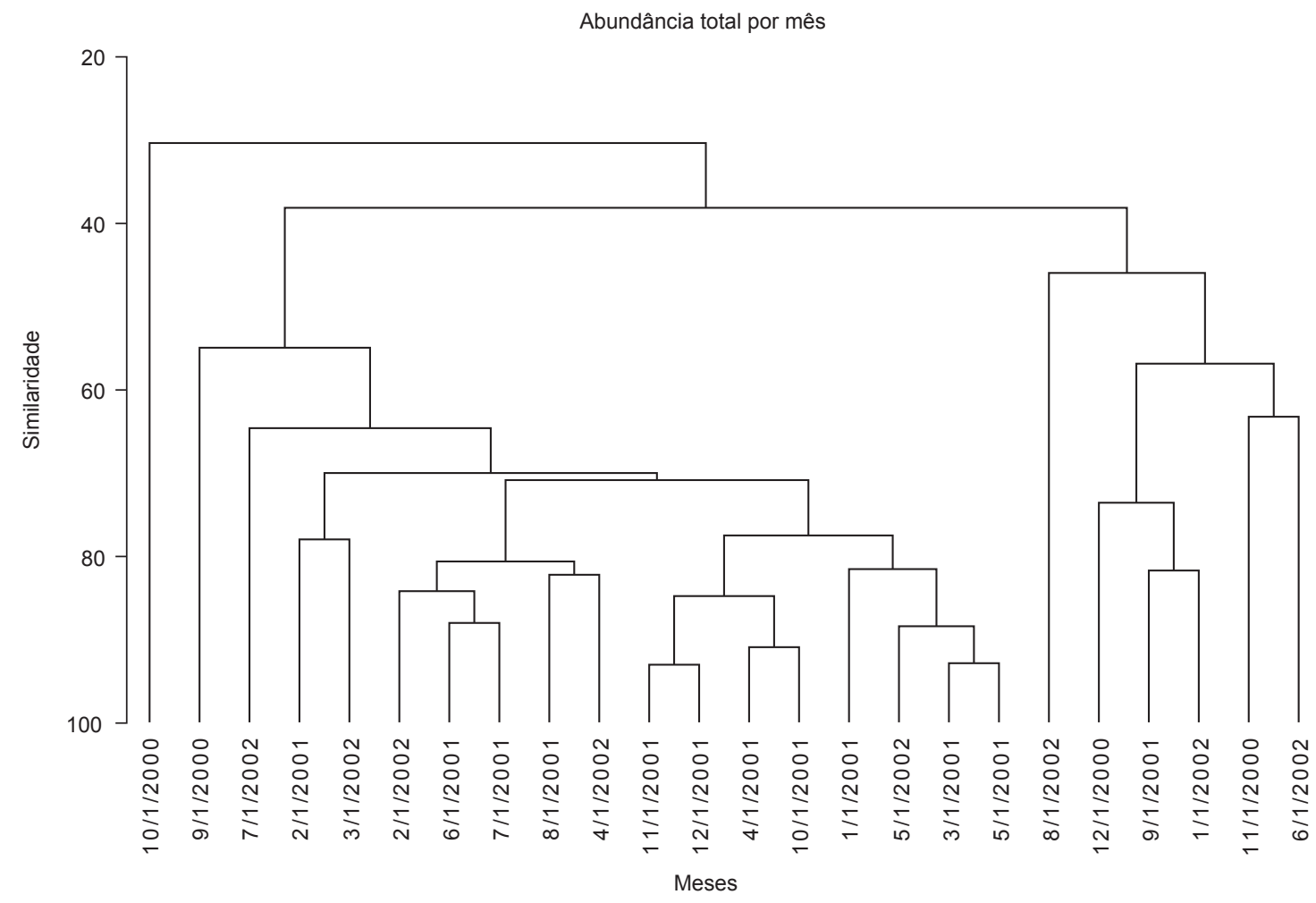

Transform: $\log (\mathrm{X}+1)$

Resemblance: S17 Bray Curtis similarity

Figura 5. Agrupamento dos períodos de coleta na região estuarina de São Vicente, baseado na abundância das espécies de bagres marinhos. No eixo X épocas no formato mês/dia/ano.

Figure 5. Clustering of the survey periods in the estuarine region of São Vicente, based on the species abundance of marine catfish. In the $\mathrm{X}$ axis, season in month/day/year format. 


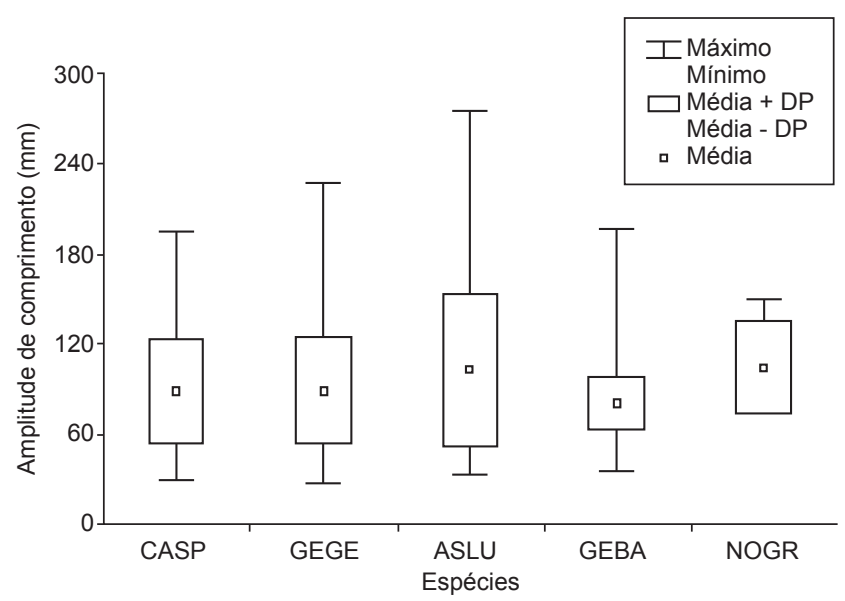

Figura 6. Comprimento padrão $(\mathrm{mm})$ das cinco principais espécies de bagres amostradas no presente estudo, na região estuarina de São Vicente, SP. $(\mathrm{CASP}=$ Cathorops spixii, $\mathrm{GEGE}=$ Genidens genidens, $\mathrm{ASLU}=$ Aspistor luniscutis, GEBA = Genidens barbus, NOGR = Notarius grandicassis).

Figure 6. Standard length $(\mathrm{mm})$ of the five marine catfish species sampled in this study, in the complex bay-estuary of São Vicente, SP. (CASP = Cathorops spixii, GEGE = Genidens genidens, $\mathrm{ASLU}=$ Aspistor luniscutis, GEBA $=$ Genidens barbus, NOGR = Notarius grandicassis .

Ao longo do período de estudo, C. spixii apresentou ampla distribuição na região estudada, sendo capturada durante praticamente todo o período amostrado, com as maiores frequiências ocorrendo no inverno de 2001 e outono de 2002. Além da sua alta abundância no estuário de São Vicente, esta espécie também foi a mais numerosa dentre todos os bagres que ocorrem em outras regiões, desde o litoral central ao sul do estado de São Paulo (Craig 1980, Mishima \& Tanji 1981, Zani-Teixeira 1983, Ribeiro Neto 1989), enquanto no litoral do Rio de Janeiro a capturada desta espécie ocorreu com menor frequiência (Araújo et al. 1998b, Pessanha et al. 2000). Segundo Paiva-Filho (1982), esta espécie utiliza a região estuarina de São Vicente como área de desova, enquanto que Zani-Teixeira (1983) e Peres-Rios (2001) sugerem que C. spixii permanece no estuário de Cananéia, durante quase todo o seu ciclo de vida.

A espécie G. genidens representou a segunda maior abundância, sendo bastante freqüente no outono e ocorrendo principalmente na região interna do estuário. Esta espécie caracterizou-se como a mais importante no estuário do rio Itanhaém, correspondendo sozinha a cerca de 50\% em número e peso (Louro 2007), com ocorrência em praticamente todos os meses e principalmente na área interna e sendo esporadicamente capturada na área marinha adjacente, segundo o autor, este estuário não foi identificado como uma área de desova, mas sim para a permanência e crescimento de jovens. Segundo Araújo (1988), esta espécie permanece na cabeçeira do estuário e interior da lagoa dos Patos (RS) e só ocasionalmente freqüenta o estuário em sua fase jovem, sem um padrão sazonal definido entre sua chegada e/ ou saída, diferentemente do observado na região de São Vicente em que há uma aparente sazonalidade para a ocorrência de indivíduos desta espécie no verão e outono.

Os valores de primeira maturação gonadal $\left(\mathrm{L}_{50}\right)$ encontrados para Cathorops spixii na região de Cananéia foram 96 e $98 \mathrm{~mm}$ (Mishima \& Tanji 1983b), 124,5 e 139 mm (Silva 1996) e, 119,2 e 106,8 mm (Peres-Rios 2001) para fêmeas e machos respectivamente. Tomando-se esses valores como indicadores da presença de jovens e adultos, observou-se o predomínio de exemplares jovens no estuário de São Vicente, uma vez que as maiores freqüências ocorreram entre os comprimentos de 64 a $85 \mathrm{~mm}$.
Para Genidens genidens os valores da primeira maturação gonadal encontrados na literatura foram $180 \mathrm{~mm}$ para as fêmeas na lagoa de Jacarepaguá (Barbieri et al. 1992) e, 133 e $160 \mathrm{~mm}$ para fêmeas e machos, respectivamente, na baía de Sepetiba (Araújo et al. 1998a). Assim, no estuário de São Vicente predominam comprimentos de 40 a $114 \mathrm{~mm}$ para esta espécie, indicando também a ocorrência de jovens.

Este padrão parece se repetir para todas as espécies: quando se considera que, para Aspistor luniscutis, no estuário do Rio Sergipe, o valor de $\mathrm{L}_{50}$ para ambos os sexos foi estimado em $170 \mathrm{~mm}$ (Alcântara 1989) e para Genidens barbus a primeira maturação gonadal para ambos os sexos foi estimada em $430 \mathrm{~mm}$ na lagoa dos Patos (Reis 1986), no estuário de São Vicente foram capturados apenas indivíduos jovens desta espécie (comprimento máximo de 196 mm). Araújo (1988) observou que G. barbus permanece no estuário durante os dois primeiros anos de vida e a partir do terceiro ano, parece deslocar-se para o interior da lagoa dos Patos, ocupando a mesma área de ocorrência dos adultos, sendo, portanto, uma espécie que apresenta ampla distribuição entre os ambientes estuarinos. Assim, o predomínio de jovens para C. spixii, G. genidens, A. luniscutis e G. barbus, indica que o estuário é área de crescimento, corroborando com os resultados de Paiva-Filho (1982) e Gurgel et al. (2000).

Não houve correlação entre os valores de salinidade da água de fundo no estuário de São Vicente e o número de exemplares. Araújo (1988) encontrou baixa correlação, embora significativa, entre a abundância dos bagres marinhos com a salinidade e a temperatura, sendo estes fatores inversamente proporcionais à abundância, e que as espécies por ele estudadas (G. genidens, G. barbus e G. planifrons) suportam uma grande variação destes fatores ambientais. Os transectos no estuário de São Vicente não cobriram completamente o gradiente salino (da região eurihalina para a oligoalina), o que pode explicar a falta de relação entre abundância dos espécimes de diferentes espécies e a salinidade.

No complexo estuarino lagunar de Cananéia, Mishima \& Tanji (1983a) capturaram o maior número de exemplares de $C$. spixii e A. luniscutis em águas com a salinidade entre 16,1 e 20 e G. genidens entre 8,1 e 12 , enquanto que, os jovens de G. barbus, B. marinus e $B$. bagre, concentram-se nas áreas de salinidade mais alta deste complexo estuarino. Essa preferência pode estar relacionada com o comportamento das formas adultas dessas espécies, que vivem em mar aberto adjacente, portanto, áreas de salinidade mais alta, migrando para as águas estuarinas na época da desova. Conseqüentemente, os bagres pequenos permanecem até atingir determinado tamanho nessa área, para depois se deslocarem para a região costeira (Mishima \& Tanji 1983a). Assim, a baixa captura destas espécies no estuário de São Vicente, principalmente $B$. bagre, pode ser explicada com base na salinidade dos ambientes e épocas estudados.

Com base no material coletado, pode-se afirmar C. spixii, G. genidens e A. luniscutis são constantes na região mais interna do estuário, C. spixii também foi constante na porção mais externa, sendo que todas as espécies de bagre capturadas ocorreram na fase juvenil pré-maturação no estuário de São Vicente, não se reproduzindo no local.

\section{Agradecimentos}

Ao Laboratório de Zoologia da Universidade de Taubaté UNITAU pelo apoio logístico. A Pró-Reitoria de Pesquisa e PósGraduação - Bolsa PIC/UNITAU. Aos colegas do Laboratório de Zoologia (UNITAU), em especial Camila L. Martins, Fernanda C. Almeida e Delma A. Ribeiro pelo auxílio nas atividades laboratoriais. A Fundação de Amparo a Pesquisa do Estado de São Paulo, Bolsa Pós-Doutorado (A.L.D.R.) (FAPESP \# 00/06505-7). À Dra Monica 
A. V. Petti e MSc Wellington S. Fernandes pelo auxílio com os testes estatísticos.

\section{Referências Bibliográficas}

ALCÂNTARA, A.Y. 1989. Ecologia da ictiofauna do estuário do rio Sergipe (estado de Sergipe, Brasil). Tese de Doutorado, Universidade de São Paulo, Instituto de Biociências, São Paulo.

ANDREATA, J.V., BARBIERI, L.R., SEBILIA, A.S.B., SILVA, M.H.C. \& SANTOS, R.P. 1989. A list of Marapendi lagoon fishes, Rio de Janeiro, Brazil. Atlântica, Rio Grande, 11:5-17.

ARAÚJO, F.G. 1984. Hábitos alimentares de três bagres marinhos (Ariidae) no estuário da lagoa dos Patos (RS), Brasil. Atlântica, Rio Grande, $7: 47-63$.

ARAÚJO, F.G. 1988. Distribuição, abundância relativa e movimentos sazonais de bagres marinhos (Siluriformes, Ariidae) no estuário da lagoa dos Patos (RS), Brasil. Rev. Bras. Zool. 5(4):509-543.

ARAÚJO, F.G., GOMES, I.D., AZEVEDO, M.C.C. \& PESSANHA, A.L.M. 1998a. Maturação e desova do bagre marinho Genidens genidens Valenciennes, 1833 (Siluriformes, Ariidae), na baía de Sepetiba, RJ. Acta Biol. Leopold. 20(1):109-122.

ARAÚJO, F.G., CRUZ-FILHO, A.G., AZEVEDO, M.C.C. \& SANTOS, A.C.A. 1998b. Estrutura da comunidade de peixes demersais da baía de Sepetiba, RJ. Rev. Bras. Biol. 458(3):417-430.

AYRES, M., AYRES Jr., M., AYRES, D.L. \& SANTOS, A.S. 2003. BioEstat 3.0: Aplicações estatísticas nas áreas das ciências biológicas e médicas. Sociedade Civil Mamirauá, CNPq, Brasília.

AZEVEDO, M.C.C., ARAÚJO, F.G., CRUZ-FILHO, A.G., GOMES, I.D. \& PESSANHA, A.L.M. 1998. Distribuição por tamanho de bagres marinhos (Siluriformes, Ariidae) na baía de Sepetiba, Rio de Janeiro. Acta Biol. Leopold., 20(2):273-288.

AZEVEDO, M.C.C., ARAÚJO, F.G., CRUZ-FILHO, A.G., GOMES, I.D. \& PESSANHA, A.L.M. 1999. Variação espacial e temporal de bagres marinhos (Siluriformes, Ariidae) na baía de Sepetiba, Rio de Janeiro. Rev. Bras. Biol. 59(3):443-454.

BARBIERI, L.R., SANTOS, R.P. \& ANDREATA, J.V. 1992. Reproductive biology of the marine catfish, Genidens genidens (Siluriformes, Ariidae), in the Jacarepaguá lagoon system, Rio de Janeiro, Brasil. Environ. Biol. Fish., 35:23-35.

CHAVES, P.T.C. 1994. A incubação de ovos e larvas em Genidens genidens (Valenciennes) (Siluriformes, Ariidae) da baía de Guaratuba, Paraná, Brasil. Rev. Bras. Zool. 11(4):641-648.

CRAIG, I.D.H. 1980. Contribuição ao conhecimento da fauna íctica costeira da região de Peruíbe, SP. I: Família Ariidae. Rev. Bras. Biol. 40(4):755-758.

CUNNINGHAM, P.T.M. 1983. Estudo comparativo da ictiofauna da costa oeste e enseada das Palmas da Ilha Anchieta, enseada do Flamengo e

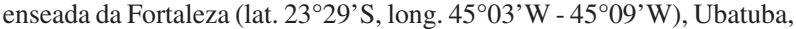
estado de São Paulo-Brasil. Tese de Doutorado, Universidade de São Paulo, Instituto Oceanográfico, São Paulo.

DAJOZ, R. 1973. Ecologia Geral. 2 ed. São Paulo: Editora da Universidade de São Paulo.

ESPÍRITO SANTO, R.V. \& ISAAC, V.J. 1999. Alimentação e aspectos da reprodução de uricica Cathorops spixii (Agassiz, 1829) (Osteichthyes, Siluriformes, Ariidae), no estuário do rio Caeté (município de BragançaPA). Bol. Mus. Para. Emílio Goeldi, ser. Zool. 15(1):95-111.

FÁVARO, L.F., FREHSE, F.A., OLIVEIRA, R.N. \& SCHWARZ JÚNIOR, R. 2005. Reprodução do bagre amarelo, Cathorops spixii (Agassiz) (Siluriformes, Ariidae), da baía de Pinheiro, região estuarina do litoral do Paraná, Brasil. Rev. Bras. Zool. 22(4):1022-1029.

FIGUEIREDO, J.L. \& MENEZES, N.A. 1978. Manual de peixes marinhos do sudeste do Brasil. II Teleostei (1). Museu de Zoologia - USP, São Paulo.

GOMES, I.D. \& ARAÚJO, F.G. 2004. Reproductive biology of marine catfishes (Siluriformes, Ariidae) in the Sepetiba bay, Brazil. Rev. Biol. Trop. 52(1):143-156.
GOMES, I.D., ARAÚJO, F.G., AZEVEDO, M.C.C. \& PESSANHA, A.L.M. 1999. Biologia reprodutiva dos bagres marinhos Genidens genidens (Valenciennes) e Cathorops spixii (Agassiz) (Siluriformes, Ariidae), na baía de Sepetiba, Rio de Janeiro, Brasil. Rev. Bras. Zool. 16(supl.2):171-180.

GURGEL, H.C.B., ALBURQUEQUE, C.Q., SOUZA, D.S.L. \& BARBIERI, G. 2000. Aspectos da biologia pesqueira em fêmeas de Cathorops spixii do estuário do rio Potengi, Natal/RN, com ênfase nos índices biométricos. Acta Scientiarum 22(2):503-505.

LOURO, M.P. 2007. A ictiofauna do estuário do rio Itanhaém, SP, Brasil: Dinâmica espaço-temporal e aspectos biológicos das espécies principais. Tese de Doutorado, Universidade de São Paulo, Instituto Oceanográfico, São Paulo.

MACIEL, N.A.L. 1995. Estudo sobre a composição, distribuição, abundância e diversidade da ictiofauna de três enseadas na região litorânea de Ubatuba - estado de São Paulo - Brasil. Dissertação de Mestrado, Universidade de São Paulo, Instituto Oceanográfico, São Paulo.

MARCENIUK, A.P. 2005. Chave para identificação das espécies de bagres marinhos (Siluriformes, Ariidae) da costa brasileira. B. Inst. Pesca, São Paulo, 31(2):89-101.

MAZZONI, R., PETITO, J. \& MIRANDA, J.C. 2000. Reproductive biology of Genidens genidens, a catfish from the Maricá lagoon, RJ. Ciência e Cultura - J. Braz. Assoc. Adv. Sci. 52(2):121-126.

MELO, S.C. \& TEIXEIRA, R.L. 1992. Distribuição, reprodução e alimentação de Cathorops spixii e Arius rugispinis (Pisces: Ariidae) do complexo Mundaú/Manguaba, Maceió-AL. Rev. Brasil. Biol. = Braz. J. Biol. 52(1):169-180.

MENEZES, N.A., BUCKUP, P.A., FIGUEIREDO, J.L. \& MOURA, R.L. 2003. Catálogo das espécies de peixes marinhos do Brasil. Museu de Zoologia da Universidade de São Paulo, São Paulo.

MISHIMA, M. \& TANJI, S. 1981. Distribuição geográfica dos bagres marinhos (Osteichthyes, Ariidae) no complexo estuarino-lagunar de Cananéia (25ㅇ, 48 W). B. Inst. Pesca, São Paulo, 8(único):157-172.

MISHIMA, M. \& TANJI, S. 1982. Nicho alimentar de bagres marinhos (Teleostei, Ariidae) no complexo estuarino-lagunar de Cananéia $\left(25^{\circ} \mathrm{S}\right.$, $48^{\circ}$ W). B. Inst. Pesca, São Paulo, 9(único):131-140.

MISHIMA, M. \& TANJI, S. 1983a. Fatores ambientais relacionados à distribuição e abundância de bagres marinhos (Osteichthyes, Ariidae) no complexo estuarino-lagunar de Cananéia $\left(25^{\circ} \mathrm{S}, 48^{\circ} \mathrm{W}\right)$. B. Inst. Pesca, São Paulo, 10(único):17-27.

MISHIMA, M. \& TANJI, S. 1983b. Maturação e desova dos bagres marinhos (Osteichthyes, Ariidae) no complexo estuarino-lagunar de Cananéia $\left(25^{\circ} \mathrm{S}\right.$, $48^{\circ}$ W). B. Inst. Pesca, São Paulo, 10(único):129-141.

PAIVA FILHO, A.M. 1982. Estudo sobre a ictiofauna do Canal de Barreiros, estuário de São Vicente, SP. Tese de livre-docência, Universidade de São Paulo, Instituto Oceanográfico, São Paulo.

PAIVA FILHO, A.M., GIANNINI, R., RIBEIRO NETO, F.B. \& SCHMIEGELOW, J.M.M. 1987. Ictiofauna do complexo baía-estuário de Santos e São Vicente, SP, Brasil. Relat. Int. Inst. Oceanogr., São Paulo, 17(1):1-10.

PERES-RIOS, E. 2001. Papel do estuário no ciclo de vida das espécies dominantes da ictiofauna do complexo estuarino-lagunar de CananéiaIguape. Tese de Doutorado, Universidade de São Paulo, Instituto Oceanográfico, São Paulo.

PESSANHA, A.L.M., ARAÚJO, F.G., AZEVEDO, M.C.C. \& GOMES, I.D. 2000. Variações temporais e espaciais na composição e estrutura da comunidade de peixes jovens da baía de Sepetiba, Rio de Janeiro. Rev. Bras. Zool. 17(1): 251-261.

PINHEIRO, P., BROADHURST, M.K., HAZIN, F.H.V., BEZERRA, T. \& HAMILTON, S. 2006. Reproduction in Bagre marinus (Ariidae) off Pernambuco, northeastern Brazil. J. Appl. Ichthyol. 22(3):189-192.

REIS, E.G. 1986. Reproduction and feeding habitats of the marine catfish Netuma barba (Siluriformes, Ariidae) in the estuary of lagoa dos Patos, Brazil. Atlântica, Rio Grande, 8:35-55. 
RIBEIRO NETO, F.B. 1989. Estudo da comunidade de peixes da baía de Santos, SP. Dissertação de Mestrado, Universidade de São Paulo, Instituto Oceanográfico, São Paulo.

RIBEIRO NETO, F.B. 1993. Análise ecomorfológica das comunidades de peixes do complexo baía-estuário de Santos e São Vicente. Tese de Doutorado, Universidade de São Paulo, Instituto Oceanográfico, São Paulo.

ROCHA, G.R.A. 1990. Distribuição, abundância e diversidade da ictiofauna na região de Ubatuba-SP (2320'S- $\left.24^{\circ} 00^{\prime} \mathrm{S}, 4^{\circ} 30^{\prime} \mathrm{W}-45^{\circ} 30^{\prime} \mathrm{W}\right)$, Brasil. Dissertação de Mestrado, Universidade de São Paulo, Instituto Oceanográfico, São Paulo.

SAUL, A.C. 1994. Comunidade ictiofaunística da ilha do Bom Abrigo, Cananéia, São Paulo, Brasil. Dissertação de Mestrado, Universidade de São Paulo, Instituto Oceanográfico, São Paulo.

SILVA, J.P. 1996. Aspectos da biologia reprodutiva de Cathorops spixii (Agassiz, 1829) das Ilhas Pai Matos (25N 4754'W) - região estuarino- lagunar de Cananéia. Dissertação de Mestrado, Universidade de São Paulo, Instituto Oceanográfico, São Paulo.

SILVA, J.P., PAIVA FILHO, A.M. \& REIS, N.S. 1998. Caracterização macro- e microscópica dos ovários do bagre amarelo, Cathorops spixii (Agassiz, 1829), durante o ciclo reprodutivo. Rev. Bras. Oceanogr. 46(2):171-185.

VELASCO, G., REIS, E.G. \& VIEIRA, J.P. 2007. Calculating growth parameters of Genidens barbus (Siluriformes, Ariidae) using length composition and age data. J. Appl. Ichthyol. 23(1):64-69.

ZANI-TEIXEIRA, M.L., 1983. Contribuição ao conhecimento da ictiofauna da baía do Trapandé, complexo estuarino-lagunar de Cananéia, SP. Dissertação de Mestrado, Universidade de São Paulo, Instituto Oceanográfico, São Paulo.

Recebido em 20/11/07

Versão reformulada recebida em 29/10/08

Publicado em 31/10/08 